\title{
eSuppl 1: Study characteristics
}

\begin{tabular}{|c|c|c|c|c|}
\hline Study (author, year) & Location & $\begin{array}{c}\text { Program } \\
\text { duration } \\
\text { (yrs) }\end{array}$ & Number of residents** & $\begin{array}{l}\text { Messick criteria } \\
\text { demonstrated }{ }^{* * *}\end{array}$ \\
\hline Abu-Laban, 2013 & $\begin{array}{l}\text { Canada (British Columbia, University of British } \\
\text { Columbia) }\end{array}$ & 5 & Participants: 12 & 2,6 \\
\hline Adler, 2011 & USA (Illinois, University of Chicago) & $\begin{array}{l}3(n=1) \\
4(n=1)\end{array}$ & $\begin{array}{l}\text { Participants across } 2 \\
\text { sites: } 69\end{array}$ & 3,4 \\
\hline Aghera, 2012* & $\begin{array}{l}\text { USA (New York, Brooklyn Maimonides Medical } \\
\text { Centre) }\end{array}$ & 3 & Participants: 15 & 1,5 \\
\hline Ahn, $2011^{*}$ & USA (Illinois, University of Chicago) & ? & not specified & 6 \\
\hline Akhtar, 2010 & USA (Florida, University of Florida) & 3 & Participants (EM): 42 & 0 \\
\hline Ali, 2013* & USA (Georgia, Emory University) & $?$ & not specified & 5 \\
\hline An-Grogan,2013* & USA (Illinois, Chicago) & $?$ & not specified & 5 \\
\hline Barlas, 2011* & USA (New York, New York Hospital Queens) & 3 & All residents: 60 & 5 \\
\hline Barsuk, 2009 & $\begin{array}{l}\text { USA (Illinois, Northwestern University Feinberg } \\
\text { School of Medicine) }\end{array}$ & 4 & $\begin{array}{l}\text { EM and IM participants: } \\
103\end{array}$ & 4 \\
\hline Beeson, 2006 & USA (Multicentre) & ? & variable & 0 \\
\hline Blouin, 2006 & Canada (Ontario, Queen's University) & 5 & All years: 30 & 0 \\
\hline Bohrn, 2014* & USA (Illinois, WellSpan York Hospital) & 3 & 21 residents & 1 \\
\hline Bounds, 2013 & USA (Multicentre) & $\begin{array}{l}3(n=3) \\
5(n=1)\end{array}$ & $\begin{array}{l}\text { PGY2 (30), } \\
\text { PGY3 (39), } \\
\text { PGY4/PGY5 (3) }\end{array}$ & 1,4 \\
\hline Brazil, 2012 & $\begin{array}{l}\text { Australia (Queensland, Royal Brisbane and } \\
\text { Women's Hospital) }\end{array}$ & 3 & All PGY1: 20 & 5,6 \\
\hline Burnette, 2009 & USA (Multicentre) & 3 & $\begin{array}{l}\text { PGY1 (37), } \\
\text { PGY2 (42), } \\
\text { PGY3 (16) }\end{array}$ & 2 \\
\hline Carrière, 2009 & Canada (Quebec, Université de Montréal) & 5 & $\begin{array}{l}\text { PGY1 (21) PGY2 (21) } \\
\text { Seniors (11) }\end{array}$ & 3 \\
\hline Chan, 2014* & Canada (Ontario, McMaster University) & 5 & PGY1 and PGY2: 14 & $1,2,3,5$ \\
\hline Christian, 2012* & USA (Illinois, Cook County Hospital Stroger) & 5 & $\begin{array}{l}\text { Participants only (45): } \\
\text { PGY2 (2), } \\
\text { PGY3 (30), } \\
\text { PGY4 (10), } \\
\text { PGY5 (3) }\end{array}$ & $3,4,6$ \\
\hline Clark, 2010* & $\begin{array}{l}\text { Canada (Vancouver, University of British } \\
\text { Columbia) }\end{array}$ & 5 & not specified & 1,2 \\
\hline Cloutier, 2013* & $\begin{array}{l}\text { USA (Portland, Oregon Health and Science } \\
\text { University) }\end{array}$ & 3 & not specified & 0 \\
\hline Cooper, 2012 & $\begin{array}{l}\text { USA (Indiana, Indiana University School of } \\
\text { Medicine) }\end{array}$ & 3 & Participants: 76 & $2,3,4$ \\
\hline Datta, 2012* & USA (New York, New York Hospital Queens) & 3 & Participants: 29 & 2,5 \\
\hline Dorfsman, 2009 & USA (Pennsylvania, University of Pittsburgh) & 3 & $\begin{array}{l}\text { Participants: PGY1 (3), } \\
\text { PGY2 (28), PGY3 (1) }\end{array}$ & $1,2,6$ \\
\hline Flowerdew, 2012 & England (London, Imperial College) & ? & not specified & 1,2 \\
\hline
\end{tabular}




\begin{tabular}{|c|c|c|c|c|}
\hline Franc, 2012 & Canada (Alberta, University of Alberta) & 5 & Participants: 25 & 0 \\
\hline Frederick, 2011 & $\begin{array}{l}\text { USA (Illinois, University of Illinois College of } \\
\text { Medicine at Peoria) }\end{array}$ & 3 & $\begin{array}{l}\text { All PGY3 (between 1994- } \\
\text { 2005): } 85 \text { (mean } 7 \text { per } \\
\text { year) }\end{array}$ & 4,5 \\
\hline Gallagher, 2013* & $\begin{array}{l}\text { USA (Illinois, Northwestern University Feinberg } \\
\text { School of Medicine) }\end{array}$ & 4 & $\begin{array}{l}\text { Participants: all } 24 \text { junior } \\
\text { residents; sample of } 6 \\
\text { senior residents }\end{array}$ & 1,2 \\
\hline Girzadas, 2007 & USA (Illinois, Advocate Christ Medical Center) & 3 & $\begin{array}{l}\text { All residents (44): } \\
\text { incoming EM1 (11) } \\
\text { EM1 (11) } \\
\text { EM2 (11) } \\
\text { EM3 (11) }\end{array}$ & $1,2,4$ \\
\hline Hauff, 2014 & USA (Michigan, University of Michigan) & 4 & Total incoming PGY1: 28 & $1,2,5$ \\
\hline Hogan, 2012* & USA (Illinois, University of Chicago) & 4 & $\begin{array}{l}\text { Participants across } 6 \\
\text { residency programs }\end{array}$ & $1,2,3$ \\
\hline Howes, 2011* & USA (Chicago) & 3 & Participants: 24 & 1,5 \\
\hline Ilgen, 2011 & USA (Boston) & 4 & Total PGY4: 15 & 1,6 \\
\hline Jang, 2013 & USA (California) & 4 & Participants: 127 & 1,2 \\
\hline Jhun, 2014* & USA & 5 & $\begin{array}{l}\text { Total residents: } 67 \\
\text { Participants: } 54\end{array}$ & $1,2,6$ \\
\hline Kassam, 2014 & Canada (Calgary) & 5 & not specified & 1,3 \\
\hline Kim, 2009 & Canada (Ottawa) & 5 & $\begin{array}{l}\text { Participants: PGY1 (32), } \\
\text { PGY3 (28) }\end{array}$ & 1,3 \\
\hline Kusmiesz, 2011* & USA (Pennsylvania) & $?$ & not specified & 4 \\
\hline Kyaw, 2012 & England (London) & 3 & not specified & $1,2,3$ \\
\hline LaMantia, 2009 & USA ( 8 centres across the country) & $\begin{array}{l}3(n=6), \\
4(n=2)\end{array}$ & not specified & $1,2,3$ \\
\hline Ledrick, 2009 & USA (Ohio) & 3 & $\begin{array}{l}\text { All residents (35): } \\
\text { PGY1 (12) } \\
\text { PGY2 (12) } \\
\text { PGY3 (11) }\end{array}$ & $1,2,3,4,5,6$ \\
\hline Ledrick, 2013* & USA (Ohio) & 3 & $\begin{array}{l}\text { Total between } 2006 \text { and } \\
\text { 2012: PGY1 (81), } \\
\text { PGY2 (83), } \\
\text { PGY3 (79) }\end{array}$ & 4 \\
\hline Lee, 2010* & Canada (Ottawa) & 5 & All PGY1: 12 & $1,3,5$ \\
\hline Lee, 2012 & USA & 3 & All PEM fellows: 240 & 2 \\
\hline Leech, 2013* & USA (Florida) & 3 & Participants: 14 & 3 \\
\hline Leone, $2011^{*}$ & USA (Oregon, Chicago) & 4 & Participants: 45 & 3 \\
\hline Lifchez, 2012 & USA (Baltimore) & 3 & Participants: 7 & 5 \\
\hline Mamtani, 2014* & USA (Pennsylvania, Philadelphia) & 4 & not specified & 0 \\
\hline Marinelli, 2012* & USA (Chicago) & 4 & Participants: 45 & 1 \\
\hline McGrath, 2014* & USA (Columbus, OH) & 3 & Participants: 35 & 5 \\
\hline McIntosh, 2012 & $\begin{array}{l}\text { USA (Jacksonville, University of Florida College } \\
\text { of Medicine, Jacksonville) }\end{array}$ & 3 & not specified & 6 \\
\hline McLaughlin, 2007 & USA (Albuquerque, New Mexico) & 3 & Participants: 27 & 2,4 \\
\hline
\end{tabular}


Canadian Medical Education Journal 2017, 8(1)

\begin{tabular}{|c|c|c|c|c|}
\hline Minnigan, 2012* & USA (Indianapolis, IN) & 3 & All PGY2: 12 & 1 \\
\hline Motov, 2011 & USA (Brooklyn, NY) & 3 & not specified & 1,2 \\
\hline Murray, 2014* & USA (Ann Arbor, Michigan) & 4 & Participants: 52 & 3 \\
\hline Nelson, 2013* & $\begin{array}{l}\text { USA (Missouri, Children's Mercy Hospital and } \\
\text { Clinic) }\end{array}$ & 3 & Participants: 55 & 5 \\
\hline Noble, 2007 & USA (Multicentre) & 4 & Participants: 12 & 2 \\
\hline Noeller, 2008 & USA (Multicentre) & 3 & All residents: 38 & 1,2 \\
\hline O'Connor, 2014* & USA (Multicentre) & ? & not specified & 0 \\
\hline Pavlic, 2014* & USA (Michigan, University of Michigan) & 4 & not specified & 1 \\
\hline Reisdorff, 2006 & USA (Michigan, Michigan State University) & 3 & $\begin{array}{l}\text { Participants: } 19: \\
\text { PGY2(9), PGY3 (10) }\end{array}$ & 1 \\
\hline Ryan, 2010 & USA (New York, New York Hospital Queens) & 3 & $\begin{array}{l}\text { All residents: } 30 \text { (10 per } \\
\text { year) }\end{array}$ & 1,3 \\
\hline Sampsel, 2014* & Canada (Ontario, University of Ottawa) & 5 & All residents: 45 & 6 \\
\hline Samuel, 2009 & Australia (multicentre) & 5 & Participants: 47 & 1 \\
\hline Scher, 2011 & $\begin{array}{l}\text { USA (Connecticut, University of Connecticut } \\
\text { Health Center) }\end{array}$ & 5 & Participants (EM): 18 & 2 \\
\hline Schwaab, 2011 & USA (Ohio, Ohio State University) & 3 & $\begin{array}{l}\text { all residents: } 36 \\
\text { participants: } 27\end{array}$ & 1,2 \\
\hline Shih, 2013* & USA (New Jersey, Morristown Medical Center) & 3 & $\begin{array}{l}\text { Total PGY1 residents } \\
\text { over } 5 \text { years: } 36 \text { (avg } 7 \\
\text { per year) }\end{array}$ & 5 \\
\hline Sullivan, 2009 & USA (Missouri, University of Missouri) & 3 & $\begin{array}{l}\text { PGY1 (10) } \\
\text { PGY2 (8) } \\
\text { PGY3 (8) }\end{array}$ & 3 \\
\hline Thundiyil, 2010 & USA (Florida, University of Florida) & 3 & $\begin{array}{l}\text { PGY3 Participants (2002- } \\
\text { 2006): } 51\end{array}$ & 5 \\
\hline Wagner, 2013* & USA (Michigan, Central Michigan University) & 3 & not specified & 2 \\
\hline Wallenstein, 2010 & USA (Georgia, Emory University) & 3 & All PGY1: 18 & 5 \\
\hline Williams, 2009 & $\begin{array}{l}\text { USA (Texas, San Antonio Military Medical } \\
\text { Center) }\end{array}$ & ? & not specified & 3 \\
\hline Wittels, 2013* & $\begin{array}{l}\text { USA (Massachusetts, Harvard Affiliated } \\
\text { Programs at Brigham and Women's Hospital } \\
\text { and Massachusetts General Hospital) }\end{array}$ & ? & not specified & 3 \\
\hline Zabar, 2009 & $\begin{array}{l}\text { USA (New York, New York University School of } \\
\text { Medicine) }\end{array}$ & ? & All PGY2: 15 & 3,6 \\
\hline
\end{tabular}

Note:

$*$ abstract only

** $=$ number of participants if number of residents unclear

*** Messick criteria: 1 = Structural validity; 2 = Content validity; $3=$ Substantive validity; $4=$ External validity; $5=$ Generalizability validity; $6=$ Consequential validity; $0=$ none reported

$\mathrm{PGY}=$ post-graduate year (i.e., residency level) 